\title{
REGULACIÓN EMOCIONAL EN JÓVENES DEPORTISTAS ANTE SITUACIONES ADVERSAS EN COMPETICIÓN
}

\author{
Cristina Guerrero Rodríguez \\ Universidad de Cádiz \\ cris.guerrero@uca.es \\ Manuel Sosa-Correa \\ Universidad Autónoma de Yucatán \\ Antonio Zayas García \\ Universidad de Cádiz \\ Rocío Guil Bozal \\ Universidad de Cádiz \\ https://doi.org/10.17060/ijodaep.2017.n1.v2.950
}

Fecha de Recepción: 10 Marzo 2017

Fecha de Admisión: 1 Abril 2017

\section{RESUMEN}

La finalidad del estudio fue examinar la regulación emocional en jóvenes deportistas, integrantes de un equipo de fútbol masculino entre 16 y 17 años, ante situaciones desfavorables en competición. Primero, se analizaron las relaciones entre diferentes aspectos psicoemocionales: Inteligencia Emocional medida con la Trait Meta-Mood Scale-24; Regulación Emocional medida con el Cognitive Emotional Regulation Questionnaire (CERQ); y Estados Emocionales medidos con el Profile of Mood States (POMS), la Rueda de Emociones de Ginebra y el Competitive State Anxiety Inventory-2 (CSAI-2). Posteriormente, tras un entrenamiento en regulación emocional mediante la técnica de Imaginación Racional Emotiva de Ellis, se realizaron los registros psicofisiológicos de la tasa cardiaca, conductancia de la piel y respiración (Biofeedback-2000 ${ }^{\mathrm{X}-\text { pert }}$ ) mientras realizaban el mismo ejercicio. El objetivo fue comprobar si los jugadores lograban regular sus emociones, ante la situación adversa, a partir del cambio en su valoración emocional cognitiva y en sus respuestas psicofisiológicas durante las diferentes fases: adaptación (A), desmotivación (D), motivación (M) y recuperación $(\mathrm{R})$. Los resultados mostraron correlaciones significativas negativas entre variables de la inteligencia emocional y los estados emocionales negativos del POMS así como con estrategias desadaptativas; y, positivas con estrategias adaptativas. Asimismo se observó que los jóvenes, tras el entrenamiento en regulación emocional, consiguieron cambiar su valoración cognitiva y su estado emocional ante la situación adversa. Esto quedó reflejado en sus respuestas cognitivas y emocionales en la Rueda y en su patrón diferencial de respuesta psicofisiológico durante las distintas fases. También en los resultados deportivos del equipo. En conclusión, el entrenamiento en regula- 


\section{REGULACIÓN EMOCIONAL EN JÓVENES DEPORTISTAS ANTE SITUACIONES ADVERSAS EN COMPETICIÓN}

ción emocional ayuda a los jóvenes a saber cómo regular sus emociones más adaptativamente, especialmente ante situaciones adversas. Esto supone una herramienta de gran utilidad para mejorar el bienestar psicológico de los deportistas y mejorar su rendimiento en competición.

Palabras clave: Regulación Emocional, Jóvenes Deportistas, Cognición, Emoción y Fisiología.

\section{ABSTRACT}

\section{Emotional regulation in young athletes facing adverse situations in competition}

This study was designed to examine the emotional regulation in a young football team (16-17 y. 0.), regarding how they face adverse situations in the competition. A sample of 21 players filled a measure of trait emotional intelligence: TMMS-24 (Fernandez-Berrocal et al., 2004, Spanish version). Emotional Cognitive Regulation was with the CERQ (Domínguez et al., 2011, Spanish version), Emotional states were assessed with the POMS (McNair et al., 1971) and the Geneva Emotional Wheel (GEW, Sacharin, Schlegel, \& Scherer, 2012), and the Competitive State Anxiety Inventory-2 (CSAl-2, Arruza et al., 2011). To increase their emotional competencies, players were training in emotional regulation with the Ellis Rational Emotive Imagination technique. The physiological variables: heart rate, skin conductance and respiration were recorded while performing the same exercise (Biofeedback-2000x-pert). Psychophysiological responses were monitored during the different phases: adaptation, motivation, motivation and recovery. Outcomes showed significant negative relationships between emotional intelligence and negative emotional states of POMS as well as with maladaptive strategies and positive with adaptive strategies. After emotional regulation training, participants were able to change their cognitive appraisals, and their emotional state in facing adverse situations as GEW measure reflected after training, and their psychophysiological parameters. Training in emotional regulation helps young football players to improve their both cognitive and emotional components of the affect and improving their competencies to face adverse situations during a football match.

Key words: Emotional Regulation, Young Athletes, Cognition, Emotion, and Physiology.

\section{ANTECEDENTES}

En los últimos años tanto la Inteligencia Emocional (IE) como la Regulación Emocional (RdE) están siendo también estudiadas en al ámbito deportivo con especial interés (Meyer \& Fletcher, 2007; Granado, Gomila \& Filella, 2013). Desarrollar la IE y RdE es clave para controlar emociones desadaptativas en el deporte, que muchas veces alteran y reducen el rendimiento y el bienestar de los deportistas, por lo que los programas para entrenar las habilidades de RdE tienen gran influencia en este ámbito (Roffé 2004).

La importancia de las emociones en el deporte se fundamenta principalmente en los estudios del afrontamiento del estrés, destacando el estrés sufrido por los deportistas en las situaciones precompetitivas (Feltz, Lirgg \& Albrecht, 1992), durante la situación competitiva (Jones \& Hardy, 1990) y/o tras la competición (Pargman, 1986). Las situaciones estresantes durante el desarrollo de las competiciones pueden producir falta de concentración, pérdida del foco atencional o incremento de la ansiedad, la frecuencia cardíaca y/o la tensión muscular (Márquez, 2004; Estrada \& Pérez, 2008). La importancia en la capacidad de RdE queda expuesta por Dominguez-Lara (2016), quien utilizando el CERQ muestra que, el manejo del estrés ayuda a la persona en sus reacciones emocionales, asociándose positivamente el componente intrapersonal con las estrategias de reinterpretación positiva y focalización en los planes. Esto indica que las personas más reflexivas tienden a utilizar estrategias más elaboradas y racionales, y menos las estrategias más automáticas como la rumiación. Así pues, y teniendo en cuenta que las emociones tienen una función adaptativa en situacio- 
nes de estrés (Folkman \& Lazarus, 1985; Frijda, 1986; Levenson, 1988) algunos estudios se han centrado en cómo las emociones positivas y negativas influyen en el afrontamiento del estrés en una situación determinada (Zautra, Affleck, Tennen, Reich \& Davis, 2005). Las emociones negativas como la ira o la ansiedad influyen negativamente sobre las relaciones sociales (Lazarus, 1999) así como sobre la aparición de posibles trastornos cardiovasculares (Palmero, Díez \& Breva, 2001) 0 de tipo afectivo (Beck, Rush, Shaw, \& Emery, 1979). Por su parte, las emociones positivas contribuyen a la resistencia y a la recuperación al estrés, al tiempo que pueden interrumpir la experiencia de emociones negativas mientras se produce la situación estresante (Fredrickson \& Joiner, 2002). La necesidad de inducir emociones positivas, y disminuir las emociones negativas, propicia el desarrollo de estrategias de RdE con el objetivo de mejorar el bienestar de los deportistas y el rendimiento deportivo.

Por otro lado, en un estudio de Balaguer, Fuentes, Meliá, García-Merita y Pérez Recio (1993), utilizando el POMS, se muestra que tanto las personas que practican deporte como las que compiten presentan mayores puntuaciones en los estados de ánimo positivos respecto a las que no practican y/o compiten.

De acuerdo con numerosos autores podemos entender la RdE como la modulación (aumentar, mantener o disminuir) de cualquier aspecto de una respuesta emocional, incluyendo experiencia y comportamiento expresivo (Gross \& John, 2003; Eisenberg \& Spinrad, 2004; Goldsmith \& Davidson, 2004). En esta línea, nuestro estudio se enmarca dentro del Modelo de RdE de Mestre y Guil (2012) entendiendo la RdE como el cambio en la valoración y/o en la activación.

Por otra parte, respecto a las múltiples maneras de actuación para cambiar las emociones ya sea mediante recursos cognitivos, conductuales o emocionales, Ellis plantea una técnica muy sencilla que es la Imaginación Racional Emotiva (Ellis \& Grieger, 1990) y que es la que hemos utilizado en esta investigación. Tiene como objetivo cambiar los sentimientos y conductas en la dirección que se considere más deseable. Aunque la RdE que plantea Ellis es a nivel intrapersonal, debe entenderse desde una concepción intra e interpersonal (Rimé, 2009), lo cual resulta especialmente necesario en los deportes de equipo ya que se produce un contagio emocional en los procesos de regulación interpersonal y ello implica, a su vez, beneficios en el afecto positivo, además de permitir establecer mayores vínculos sociales.

Considerando la importancia de las emociones y su regulación en el desempeño deportivo, se plantea esta investigación para conocer los aspectos emocionales de los jugadores y así, entrenarlos para que sepan regular las emociones que estén siendo desadaptativas para sus fines por otras más adaptativas.

\section{OBJETIVOS DE LA INVESTIGACIÓN}

El principal objetivo del estudio ha sido explorar la regulación emocional en jóvenes deportistas cuando se enfrentan a situaciones adversas o desfavorables en competición.

A partir de aquí, nos planteamos los siguientes objetivos específicos:

(1) examinar la relación existente entre las variables de inteligencia emocional, uso de determinadas estrategias de regulación emocional y estados emocionales,

(2) comprobar si el entrenamiento en regulación emocional ha sido efectivo para alcanzar un cambio en la valoración emocional cognitiva de la situación adversa y si queda reflejado en un patrón diferencial de respuesta fisiológica.

\section{Participantes}

La muestra la conformaron los integrantes de un equipo masculino de fútbol juvenil de la provincia de Cádiz, con edades comprendidas entre los 16 y los 17 años $(M=16.36$; $D T=.48)$. Todos 


\section{REGULACIÓN EMOCIONAL EN JÓVENES DEPORTISTAS ANTE SITUACIONES ADVERSAS EN COMPETICIÓN}

Ios que participaron lo hicieron de forma voluntaria. De los 25 jugadores, entre titulares y suplentes que componen el equipo, finalmente cuatro de ellos no pudieron participar por estar lesionados con lo cual la muestra final del estudio la compusieron 21 jugadores.

\section{METODOLOGÍA E INSTRUMENTOS}

La presente investigación fue motivada a partir de la solicitud del equipo técnico que dirige y entrena al equipo de fútbol en cuestión, concretamente por la situación de desmotivación y estados emocionales negativos que presentaban los jugadores ante los resultados adversos en competición. Así, tras la primera toma de contacto y entrevista con el equipo técnico se comenzó el estudio.

La recogida de datos se llevó a cabo en el lugar donde entrena habitualmente el equipo de fútbol. En primer lugar, se realizó una presentación para que los jugadores, así como el resto del equipo técnico, conocieran los objetivos principales que perseguía el estudio. Una vez se les había informado sobre todo el procedimiento para participar en la investigación, se les administró el consentimiento informado para que lo firmaran sus los tutores legales. Seguidamente, se concretaron las fechas para la administración de las pruebas y la realización de los ejercicios del entrenamiento en RdE.

\section{Procedimiento}

Como se ha señalado, en un primer momento se llevó a cabo el análisis de la problemática del equipo. Posteriormente se administraron los cuestionarios y, en otra sesión, se presentó de manera simplificada el modelo ABC de Albert Ellis (Ellis, \& Abrahms, 2005) con el objetivo de mostrar la importancia del pensamiento, respecto a sus emociones y conductas, precisamente ante una situación adversa.

En las siguientes sesiones se llevó a cabo el entrenamiento en regulación emocional basado en Ios ejercicios de Imaginación Racional Emotiva de Ellis (Grieger \& Marañón, 1990) que tiene como objetivo que la persona aprenda a cambiar sus sentimientos y conductas en la dirección que considere más deseable. Concretamente, se les pedía que visualizaran una situación adversa que les generase desmotivación e identificaran sus pensamientos y conductas para, posteriormente ante esa misma situación, identificaran aquellos pensamientos y conductas que creyesen que podían ayudarles a retomar la motivación, cambiando así su valoración y su estado emocional.

Tras comprobar que habían entendido los ejercicios, y que podían pasar de la desmotivación a la motivación a voluntad, se les instó para los practicaran en sus casas.

Por último, se realizaron las sesiones de registros psicofisiológicos en las que se medía la tasa cardiaca (TD), el nivel de conductancia de la piel (SCL) y la frecuencia respiratoria (RESP) en cada uno de los participantes. Esto se llevó a cabo mientras realizaban el mismo ejercicio de RdE que se les había enseñado y habían practicado. Asimismo, mediante la Rueda de Emociones, se registró el estado emocional que los participantes sentían tanto antes de comenzar la sesión, como durante y después de la realización de las dos tareas de visualización (situación de desmotivación y motivación). Concretamente, tenían que señalar aquella emoción que predominase en cada ocasión e indicar su intensidad (de 1 a 5, de menor a mayor) para valorar así, si en general, la realización de cada una de las tareas era percibida como agradable o desagradable (dimensión de valencia). Además, la rueda de Ginebra también proporciona información acerca del nivel de activación del organismo (dimensión de arousal) según se sitúe la persona en los cuadrantes superiores o inferiores.

\section{Instrumentos}

En un primer momento se llevó a cabo una entrevista semiestructurada para recoger algunos datos personales, hábitos de sueño y alimentación, salud, etc. Posteriormente, se administraron los siguientes cuestionarios. 
El Trait Meta-Mood Scale, para medir la inteligencia emocional percibida (TMMS-24) en su versión española (Fernandez-Berrocal y cols., 2004). Consta de 24 ítems con 3 subescalas de 8 ítems cada una: Atención (At), Claridad (Cl) y Reparación (Re) con respuestas tipo Likert de 5 puntos (1=Nada de acuerdo a $5=$ Totalmente de acuerdo). La fiabilidad para cada subescala fue: 0.80 Atención, 0.81 Claridad y 0.81 Reparación, y la total de 0.89 .

El Competitive State Anxiety Inventory 2 reducido (CSAl-2) en su versión española (ArruzaGabilondo y cols., 2012). Consta de 27 ítems y tres subescalas con 9 ítems cada una. La escala de respuesta tipo Likert va de 1 (nada) a 4 (mucho). La fiabilidad de las subescalas fue de 0.90 Ansiedad Somática, 0.87 Ansiedad Cognitiva y 0.93 Autoconfianza.

La prueba Profile of Mood States (POMS) en su versión española (Balaguer, Fuentes, Meliá, García-Merita y Pérez-Recio, 1993) para medir estados emocionales. Compuesta por 58 adjetivos y 6 factores con las siguientes fiabilidades: 0.92 Depresión, 0.86 Cólera, 0.80 Vigor, 0.83 Fatiga, 0.86: Tensión, 0.80 Confusión y 0.84 el Total.

La prueba Cognitive Emotion regulation Questionnaire (CERQ) para medir estrategias de regulación emocional cognitiva en su versión española (Domínguez-Sánchez, Lasa, Amor y Holgado, 2013). Mide 9 estrategias de regulación cognitiva de las emociones, algunas consideradas más adaptativas que otras. La escala de respuesta es tipo Likert (1=casi nunca a $5=$ casi siempre). Los valores de consistencia interna están comprendidos entre 0,68 y 0,83 (Garnefski \& Kraaij, 2007).

La prueba Geneva Emotion Wheel (GEW; Sacharin, Schlegel, \& Scherer, 2012), o Rueda de Emociones en español, es un instrumento desarrollado por el Grupo de investigación de Ginebra, liderado por Klaus Scherer, para medir la valoración emocional. Supone un método de medición de la experiencia emocional de elección forzosa desde un enfoque dimensional. Es una forma gráfica, sencilla y directa para medir el estado emocional y su intensidad.

Finalmente, para el registro de las variables fisiológicas (TC, SCL y RESP) se utilizó el sistema Biofeedback-2000X-pert. Es un sistema modular inalámbrico que permite registrar de forma muy cómoda diferentes parámetros fisiológicos ya que se conecta a través de Bluetooth. Concretamente utilizamos los módulos: "Modul MULTI" para la conductancia de la piel (SCL), la tasa cardiaca (TC) y "Modul RESP" para la frecuencia respiratoria.

\section{RESULTADOS}

El análisis de los datos se realizó mediante el programa estadístico SPSS (v.22). Para una primera aproximación se realizó un análisis de tipo descriptivo. A continuación se presentan las medias y desviaciones típicas obtenidas en las variables psicoemocionales medidas.

Tabla 1

Medias y Desviaciones Típicas (DT) para las subescalas de la TMMS-24 y CSAI-2 ( $n=21)$

\begin{tabular}{lccllccc}
\hline TMMS-24 & Media & DT & & CSAI-2 & Media & DT \\
\hline Atención & 24.44 & $(7.44)$ & & Ansiedad Cognitiva & 56.38 & $(13.83)$ \\
Claridad & 27.44 & $(6.97)$ & & Ansiedad Somática & 43.51 & $(15.42)$ \\
Reparación & 27.78 & $(6.74)$ & & Autoconfianza & 72.59 & $(17.17)$ \\
\hline
\end{tabular}


Tabla 2

Medias y Desviaciones Típicas (DT) para las subescalas del POMS y CERQ $(n=21)$

\begin{tabular}{|c|c|c|c|c|c|}
\hline POMS & Media & DT & CERQ & Media & DT \\
\hline Tensión & 14,09 & $(6,91)$ & Autoculpa & 10.38 & $(2.55)$ \\
\hline Depresión & 10,75 & $(9,40)$ & Rumiación & 13.23 & (3.89) \\
\hline Cólera & 14,08 & $(8.88)$ & Focalización Positiva & 12.57 & $(3.34)$ \\
\hline Vigor & 19,80 & $(5,02)$ & Focalización en Planes & 13.15 & (3.63) \\
\hline Fatiga & 9,63 & (7.88) & Reevaluación Positiva & 14.28 & $(4.27)$ \\
\hline Confusión & 7,90 & $(5.63)$ & Catastrofización & 15.14 & $(3.24)$ \\
\hline
\end{tabular}

A partir de estos resultados, y teniendo en cuenta los baremos correspondientes, vemos que las puntuaciones de la muestra se hallan dentro de los valores medios.

Posteriormente, para analizar el grado de asociación entre las diferentes variables, y dar respuesta al objetivo (1), se utilizó el coeficiente de correlación de Spearman. En la tabla 3 podemos observar que es la subescala de Reparación emocional de la TMMS-24 la que correlaciona significativamente y de forma negativa con todos los estados negativos del Perfil de Estados de Ánimo. Por su parte, esta subescala también es la única que correlaciona, en este caso positivamente, con la Autoconfianza.

Tabla 3

Correlaciones de Spearman entre las subescalas de la TMMS-24, POMS y CSAI-2

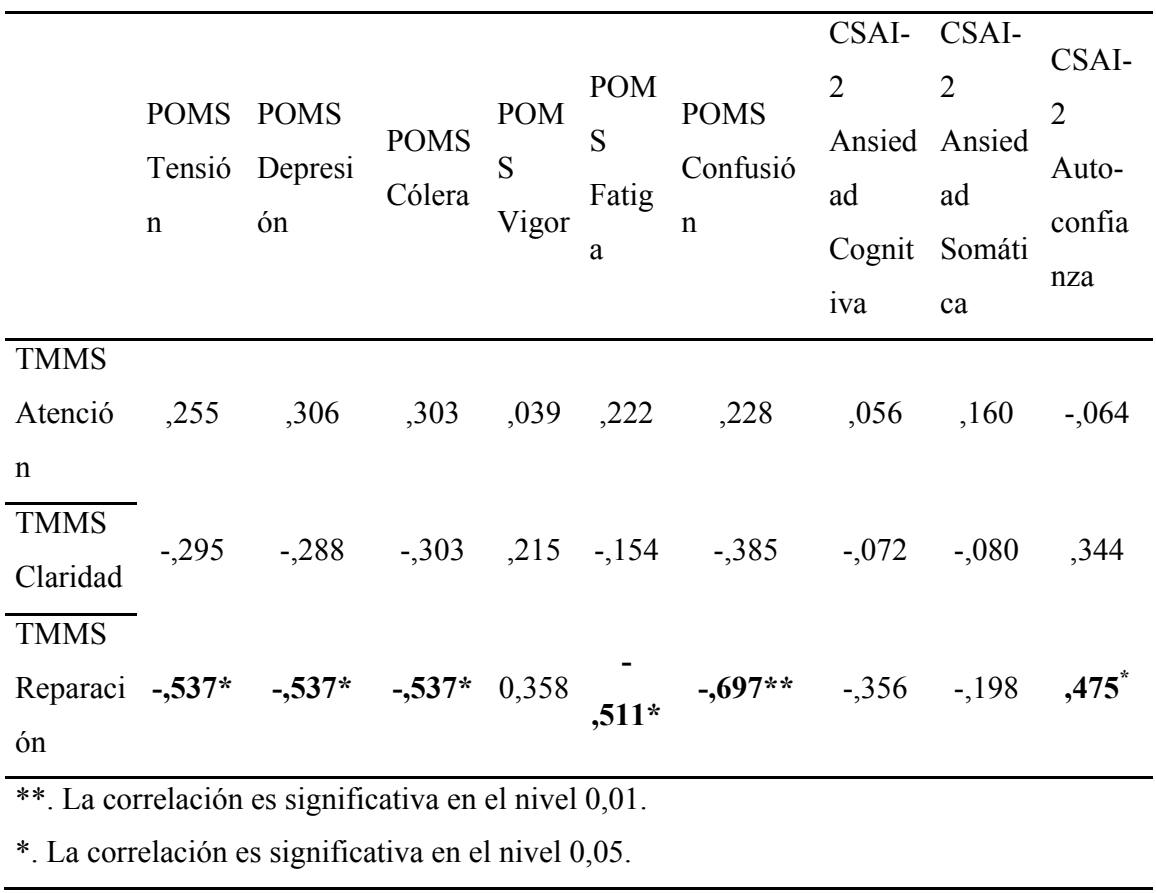


Y, en la tabla 4, vemos que nuevamente que es la subescala de Reparación (TMMS-24) la que correlaciona significativa y positivamente con las estrategias adaptativas de Aceptación y Focalización en Planes, y negativamente con la Catastrofización y Culpar a Otros menos adaptativas.

Por su parte los estados de ánimo negativos del POMS de Tensión, Depresión, Fatiga y Confusión son los que muestran correlaciones significativas negativas con la estrategia adaptativa de Focalización en Planes, y la Tensión además con Focalización Positiva. En cuanto a las correlaciones positivas, el estado de Depresión el que se relaciona con la estrategia menos adaptativa de Culpar a Otros y la Confusión con la Catastrofización.

Por último, observamos que la Ansiedad Cognitiva se relaciona significativa y negativamente con las estrategias adaptativas de Focalización en Planes y Puesta en Perspectiva; mientras que la Autoconfianza se relaciona significativa y positivamente con las estrategias adaptativas de Focalización en Planes, Reevaluación Positiva y Puesta en Perspectiva; sin embargo, también correlaciona positivamente con la Rumiación que es considerada menos adaptativa.

Tabla 4

Correlaciones de Spearman entre las subescalas de la TMMS-24, POMS, CSAI-2 y las estrategias de RdE del CERQ

\begin{tabular}{|c|c|c|c|c|c|c|c|c|c|}
\hline & $\begin{array}{c}\text { Aut } \\
\text { ocul } \\
\text { pa }\end{array}$ & $\begin{array}{l}\text { Acept } \\
\text { a-ción }\end{array}$ & $\begin{array}{l}\text { Rumi } \\
\text { a- } \\
\text { ción }\end{array}$ & $\begin{array}{c}\text { Focaliza } \\
\text { ción } \\
\text { Positiva }\end{array}$ & $\begin{array}{l}\text { Focaliza } \\
\text { ción en } \\
\text { Planes }\end{array}$ & $\begin{array}{c}\text { Reevalua } \\
\text { ción } \\
\text { Positiva }\end{array}$ & $\begin{array}{c}\text { Puesta en } \\
\text { perspercti } \\
\text { va }\end{array}$ & $\begin{array}{l}\text { Catastr } \\
\text { o- } \\
\text { fizació } \\
\text { n }\end{array}$ & $\begin{array}{l}\text { Culpar } \\
\text { a Otros }\end{array}$ \\
\hline $\begin{array}{l}\text { TMMS } \\
\text { Atenció } \\
n\end{array}$ & $\begin{array}{c}- \\
, 240\end{array}$ &,- 088 & ,225 &,- 403 &,- 239 & ,160 &, 170 & ,383 & 202 \\
\hline $\begin{array}{l}\text { TMMS } \\
\text { Claridad }\end{array}$ & $\begin{array}{c}- \\
, 096\end{array}$ & ,303 & ,289 & 0,000 & ,263 & ,240 &,- 009 & ,364 & ,246 \\
\hline $\begin{array}{l}\text { TMMS } \\
\text { Reparaci } \\
\text { ón }\end{array}$ & ,285 & ,435" & ,215 & ,254 &, $569^{* *}$ & ,277 & ,211 &,$- 515^{*}$ &,$- 524^{*}$ \\
\hline $\begin{array}{l}\text { POMS } \\
\text { Tensión }\end{array}$ & $\begin{array}{c}- \\
, 012\end{array}$ &,- 149 &,- 028 &,$- 483^{*}$ &,$- 509^{*}$ &,- 373 &,- 172 & , 171 & ,120 \\
\hline $\begin{array}{l}\text { POMS } \\
\text { Depresió } \\
\mathrm{n}\end{array}$ & $\begin{array}{c}- \\
, 244\end{array}$ &,- 160 &,- 205 &,- 310 &,$- 680^{* *}$ &,- 144 &,- 007 & ,380 &, $495^{*}$ \\
\hline $\begin{array}{l}\text { POMS } \\
\text { Cólera }\end{array}$ & ,110 & ,146 & ,056 &,- 071 &,- 258 &,- 069 & ,079 & ,242 & ,220 \\
\hline $\begin{array}{l}\text { POMS } \\
\text { Vigor }\end{array}$ & ,092 & ,025 & ,215 &,- 116 & ,165 &,- 039 &,- 099 &,- 358 &,- 398 \\
\hline
\end{tabular}




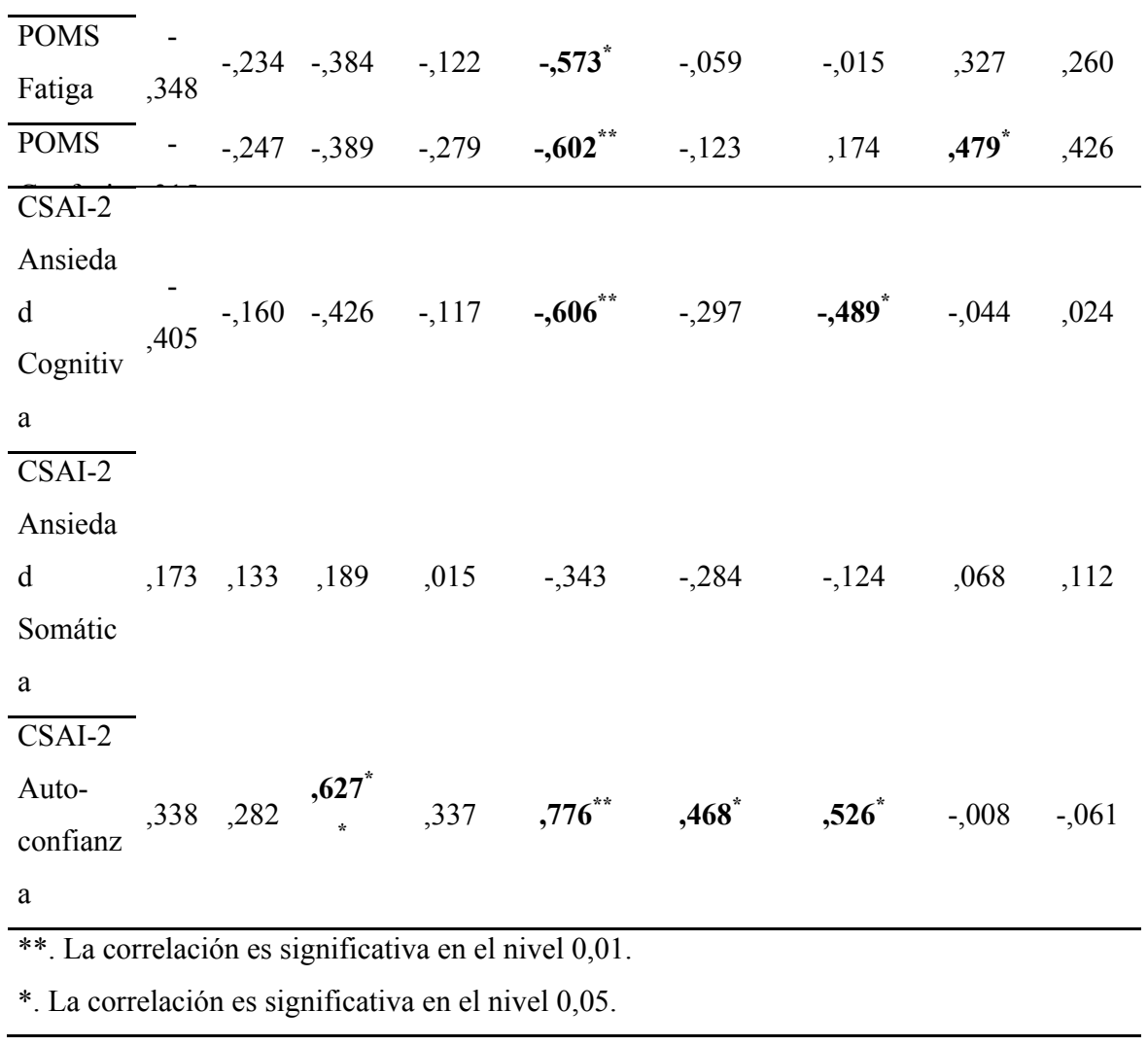

A partir de las puntuaciones medias de las variables psicofisiológicas en las diferentes fases se realizaron las correspondientes comparaciones mediante la prueba de Wilcoxon. En la tabla 5 se muestran los resultados.

En cuanto a la TC vemos que las mayores diferencias significativas se dan entre las fases de tarea tanto de Motivación como Desmotivación respecto a la de Recuperación, lo que indica que se produjo un cambio en la activación de los participantes de las fases tarea de RdE a las de las fases de recuperación (reposo). En cuanto al nivel de conductancia apreciamos que la mayor diferencia significativa se da entre la fase de Adaptación y la tarea de Motivación, lo que muestra también un cambio en la activación en el sentido que acabamos de comentar. $Y$ en cuanto a la frecuencia respiratoria vemos que las diferencias significativas se dan entre la fase de Adaptación en relación a las de tarea de Desmotivación y Motivación así como de Recuperación. 
Tabla 5

Comparación de medias de las respuestas psicofisiológicas registradas

\begin{tabular}{ccccccc}
\hline & TC_A - & TC_A - & TC_A - & TC_D - & TC_R - & TC_R - \\
& TC_D & TC_M & TC_R & TC_M & TC_D & TC_M \\
\hline Z & $-2,456^{\mathrm{b}}$ & $-1,900^{\mathrm{b}}$ &,$- 435^{\mathrm{c}}$ &,$- 816^{\mathrm{c}}$ & $-2,142^{\mathrm{c}}$ & $-2,202^{\mathrm{c}}$
\end{tabular}

Asymp.

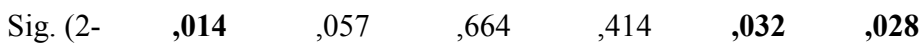

tailed)

\begin{tabular}{ccccccc}
\hline & SCL_A - & SCL_A - & SCL_A - & SCL_D - & SCL_R - & SCR_R - \\
& SCL_D & SCL_M & SCL_R & SCL_M & SCL_D & SCR_M \\
\hline Z &,$- 628^{\mathrm{b}}$ & $-2,223^{\mathrm{b}}$ & $-2,845^{\mathrm{b}}$ & $-2,578^{\mathrm{b}}$ & $-2,934^{\mathrm{b}}$ & $-1,481^{\mathrm{c}}$
\end{tabular}

Asymp.

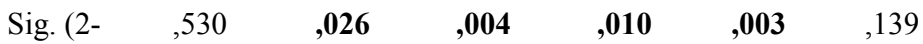

tailed)

\begin{tabular}{ccccccc}
\hline & RESP_A & RESP_A & RESP_A & RESP_M & RESP_R & RESP_R \\
& - & - & - & - & - & - \\
& RESP_D & RESP_M & RESP_R & RESP_D & RESP_D & RESP_M \\
\hline Z & $-2,430^{\mathrm{c}}$ & $-2,514^{\mathrm{c}}$ & $-2,284^{\mathrm{c}}$ & $-1,065^{\mathrm{c}}$ &,$- 976^{\mathrm{c}}$ &,$- 850^{\mathrm{c}}$
\end{tabular}

Asymp.

Sig. (2-

,015

,012

, 022

,287

,329

,395

tailed)
a. Wilcoxon Signed Ranks Test
b. Based on negative ranks.
c. Based on positive ranks.

Un dato que cabe destacar es la ausencia de diferencias significativas entre las fases de tarea de Desmotivación y Motivación (excepto en SCL, pero es mínima) que se explica porque la gran parte de los participantes, aunque realizó el cambio de valoración emocional cognitiva (de izquierda a derecha), siguió posicionándose en los mismos cuadrantes superiores o inferiores de la rueda con lo cual la activación seguía siendo similar.

Finalmente, para dar respuesta al objetivo (2) referido al cambio de valoración emocional cognitiva, se pudo comprobar a partir del posicionamiento de cada participante en la Rueda de Emociones, así como por sus respuestas escritas sobre los pensamientos que tuvieron en cada una de las dos fases de tarea (Desmotivación y Motivación), cómo todos ellos pasaron de los cuadrantes izquierdos (Ira, n=7; Tristeza, $n=3$; Decepción, $n=9$; Desprecio, $n=1$; y Culpable $n=1$ ) a los cuadrantes derechos de la rueda (Alegre, $n=8$; Satisfecho, $n=5$; Orgulloso de sí $n=5$; y Alertado, $n=3$ ). 


\section{CONCLUSIONES}

Tal y como se ha visto, cada vez son más los expertos que insisten en la relevancia de que el deporte se estudie desde una perspectiva integradora, considerando tanto aspectos físicos, técnicos y tácticos como psicológicos. En este contexto, no debemos olvidar la conexión que se produce entre la emoción, la cognición y la fisiología y como esto influye en el ámbito deportivo también. A partir de la bibliografía revisada y de los resultados presentados en esta investigación se recalca la importancia de estudiar e incorporar el componente emocional en este campo también, tanto en los entrenamientos como, especialmente, en la competición para mejorar el rendimiento (Rimé, 2009).

Se ha comprobado como un breve entrenamiento en RdE con jóvenes deportistas ha ayudado a, por un lado, que sean más conscientes de sus estados emocionales, atender a los mismos y discriminar su intensidad; y, por otro lado, a ser capaces de cambiar su valoración emocional cognitiva ante una situación adversa, que era negativa por otra de carácter positivo, al tiempo que afrontar así dicha situación de una forma mucho más adaptativa. En relación a este último aspecto, también se ha dejado constancia de la relación existente entre el uso de estrategias de RdE más adaptativas con la presencia de unos estados emocionales más positivos, lo cual a su vez enfatiza la importancia de desarrollar programas de RdE (Granado, Gomila \& Filella, 2013).

Resumiendo, conseguir que los deportistas sean conscientes de sus emociones, así como de su intensidad y que desarrollen la capacidad de RdE, mediante el uso de estrategias más adaptativas, repercutirá positivamente sobre su bienestar y redundará en una mejor práctica y competición deportiva.

\section{REFERENCIAS BIBLIOGRÁFICAS}

Arruza Gabilondo, J.A., Palacios Moreno, M., González Rodríguez, 0., Cecchini Estrada, J.A., \& Arribas Galarraga, S. (2012). Validación del competitive state anxiety inventory 2 reducido (csai2 re) mediante una aplicación web. Revista Internacional de Medicina y Ciencias de la Actividad Física y del Deporte, sin mes, 539-556.

Balaguer, I., Fuentes, I., Meliá, J.L., García-Merita, M., \& Pérez Recio, G. (1993). El perfil de los estados de ánimo (POMS). Revista de Psicología del deporte, 2(2), 0039-52.

Beck, A.T., Rush, A.J., Shaw, B.F., \& Emery G. (1979). Cognitive therapy of depression. New York: Guilford Press.

Caprara, G.V., Barbaranelli, C., \& Borgogni, L. (1995). BFQ. Cuestionario "Big Five”. Manual. Madrid: TEA Ediciones.

Domínguez-Lara, S. (2016). Inteligencia emocional y estrategias cognitivas de regulación emocional en universitarios de Lima: un análisis preliminar. Revista del Hospital Psiquiátrico de La Habana, 13(2), [aprox. 5 p.].

Domínguez-Sánchez, F.J., Lasa-Aristu, A., Amor, P.J., \& Holgado-Tello, F.P. (2013). Cognitive Emotion Regulation Questionnaire (CERQ). Psychometric properties of the Spanish version. Assessment, 20(2), 253-261.

Ellis, A., \& Abrahms, E. (2005). Terapia Racional Emotiva (2ª edición). México: Editorial Pax.

Ellis, A., \& Grieger, R. (1990). Manual de terapia racional-emotiva (Vol. 2). Bilbao: Desclée de Brouwer.

Eisenberg, N., \& Spinrad, T.L. (2004). Emotion-related regulation: Sharpening the definition. Child Development, 75, 334-339.

Feltz, D.; Lirgg, C., \& Albrecht, R. (1992). Psychological implications of competitive running in elite young distance runners: A longitudinal analysis. The Sport Psychologist, 6, 128-138.

Fernandez-Berrocal, P., Extremera, N., \& Ramos, N. (2004). Validity and reliability of the Spanish 
modified version of the Trait Meta-Mood Scale. Psychological Reports, 94, 751-755.

Folkman, S., \& Lazarus, R.S. (1985). If it changes it must be a process: study of emotion and coping during three stages of a college examination. Journal of personality and social psychology, 48(1), 150.

Fredrickson, B. L., \& Joiner, T. (2002). Positive emotions trigger upward spirals toward emotional well-being. Psychological science, 13(2), 172-175.

Frijda, N.H. 1986. The emotions, Cambridge, UK: Cambridge University Press.

Garnefski, N. \& Kraaij, V. (2007). The Cognitive Emotion Regulation Questionnaire: Psychometric features and prospective relationships with depression and anxiety in adults. European Journal of Psychological Assessment, 23, 141-149.

GEW; available under Research Tools at http://www.unige.ch/fapse/emotion

Gimeno, E.C., Ruano, F.J.S.R., Jiménez, R., Fenoll, A.N., \& García, T. (2002). Motivación y ansiedad en jugadores de tenis. European Journal of Human Movement, 9, 141-161.

Goldsmith, H.H., \& Davidson, R.J. (2004). Disambiguating the components of emotion regulation. Child Development, 75, 361-365.

Granado, X., Gomila, M., \& Filella, G. (2013). Regulación emocional de los resultados adversos en competición. Estrategias funcionales en deportes colectivos. Cuadernos de Psicología del Deporte, 14 (1), 63-72.

Gross, J.J., \& John, O.P. (2003). Individual differences in two emotion regulation processes: Implications for affect, relationships, and well-being. Journal of Personality and Social Psychology, 85,348-362.

Hardy, L., Mullen, R., \& Jones, G. (1996). Knowledge and conscious control of motor actions under stress. British Journal of psychology, 87(4), 621-636.

Hodges, N.J. \& Starkes, J.L. (1996). Wrestling with the nature of expertise: A sportspecific test of Ericsson, Krampe \& Tesch-Römer's (1993) theory of 'deliberate practice'. International Journal of Sport Psychology, 27, 400-424.

Isen, A.M., Rosenzweig, A.S., \& Young, M.J. (1991). The influence of positive affect on clinical problem solving. Medical Decision Making, 11(3), 221-227.

Jones, G., \& Hardy, L. (1990). Stress in sport: Experiences of some elite performers. In G. Jones \& L. Hardy (Eds.), Stress and performance in sport (pp. 247-277). Chichester: John Wiley.

Lazarus, R.S. (1999). Stress and emotion: A new synthesis. London: Free Association Books.

Levenson, R.W. (1988). Emotion and the autonomie nervous system: A prospectus for research on autonomie specificity. En H.L. Wagner, Social psychophysiology and emotion: Theory and clinical applications, pp. 17-41. London: Wiley

Mestre, J.M. y Guil, R. (2012). Regulación de emociones. Una vía a la adaptación personal y social. Madrid: Pirámide.

Meyer, B.B., \& Fletcher, T.B. (2007). Emotional intelligence: A theoretical overview and implications for research and professional practice in sport psychology. Journal of Applied Sport Psychology, 19(1), 1-15.

Palmero, F., Díez, J.L., \& Breva Asensio, A. (2001). Type A behavior pattern today: Relevance of the JAS-S factor to predict heart rate reactivity. Behavioral Medicine, 27(1), 28-36.

Pargman, D. (1986). Stress and motor performance: Understanding and coping. Tallahassee, FL.

Rimé, B. (2009). Emotion elicits the social sharing of emotion: Theory and empirical review. Emotion Review, 1(1), 60-85.

Roffé, M., \& Ucha, F.E.G. (Eds.). (2004). Alto rendimiento: psicología del deporte: tendencias actuales. Lugar:.Buenos Aires. 
Ruiz-Juan, F., Zarauz, A., \& Flores-Allende, G. (2015). Percepción del éxito en corredores de fondo en ruta en función de variables socio-demográficas. Retos, 27, 136-139.

Sacharin, V., Schlegel, K., \& Scherer, K.R. (2012). Geneva Emotion Wheel rating study (Report). Geneva, Switzerland: University of Geneva, Swiss Center for Affective Sciences.

Scherer, K.R. (2005). ¿Qué son las emociones? ¿Y cómo pueden ser medidas? Social Science Information, 44(4), 695-729.

Zautra, A.J., Affleck, G.G., Tennen, H., Reich, J.W., \& Davis, M.C. (2005). Dynamic approaches to emotions and stress in everyday life: Bolger and Zuckerman reloaded with positive as well as negative affects. Journal of personality, 73(6), 1511-1538. 